\title{
LOW TEMPERATURE TRANSPORT AND MAGNETIC PROPERTIES OF $\mathrm{SmB}_{6}$
}

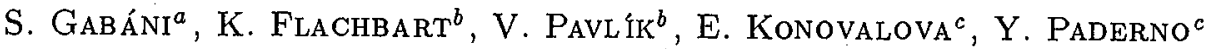 \\ AND T. HERRMANNSDÖRFER ${ }^{d}$ \\ ${ }^{a}$ Department of Experimental Physics, P.J. Safárik University \\ Park Angelinum 9, 04154 Košice, Slovakia \\ ${ }^{b}$ Institute of Experimental Physics, SAS, 04353 Košice, Slovakia \\ 'Institute for Problems of Materials Science, UAS, $252680 \mathrm{Kiev}$, Ukraine \\ ${ }^{d}$ Physikalisches Institut, Universität Bayreuth, 95440 Bayreuth, Germany
}

We present results of transport and magnetic properties of three single-crystalline samples of the intermediate valence small-gap semiconductor $\mathrm{SmB}_{6}$ at low temperatures. The received resistivity dependences of the samples below $0.5 \mathrm{~K}$ exhibit an activated behavior with an energy gap of a few $\mathrm{mK}$. The temperature dependences of the magnetic susceptibility show an increase below $15 \mathrm{~K}$ which can be accounted for by impurities, by bare $\mathrm{Sm}^{3+}$ ions or by a small amount of in-gap magnetic $4 f^{5} 5 d^{1}$ states.

PACS numbers: $75.20 . \mathrm{Hr}, 71.28 .+\mathrm{d}$

Samarium hexaboride is a typical representative of intermediate valence semiconductors and is considered as an example of a Kondo insulator [1-4]. Almost three decades after the first investigations on this material [5], a large number of detailed and comprehensive studies of $\mathrm{SmB}_{6}$ have been performed. However, many fundamental properties of $\mathrm{SmB}_{6}$ such as the electrical conductivity and the behavior of magnetic susceptibility at lower temperatures are not fully understood. The resistivity $\rho(T)$ is slightly temperature dependent down to $50 \mathrm{~K}$ below which it starts to increase exponentially $[1,5]$ due to the opening of a hybridization gap in the density of states. Between $15 \mathrm{~K}$ and $5 \mathrm{~K}$ it can be described by an exponential increase $\rho(T) \propto \exp \left(-\Delta_{0} / k_{\mathrm{B}} T\right)$, with an activation energy $\Delta_{0}$ of a few $\mathrm{meV}[1,5-9]$. However, there exists disagreement about the temperature dependence of $\rho$ below about $4 \mathrm{~K}$ and about the origin of the observed residual conductivity. The residual conductivity has been interpreted in terms of the minimum conductivity [7], as a Wigner lattice formation [9], or attributed to surface states [10]. Thermally activated behavior and hopping transport have been proposed to account for the temperature dependence $[5,9]$ in this temperature range. The magnetic susceptibility reveals the characteristic features of an intermediate valence compound: a Curie-Weiss like susceptibility at temperatures above $100 \mathrm{~K}$ indicating a local-moment behavior, and a temperature independent susceptibility at low temperatures due to a nonmagnetic $4 f^{5} 5 d^{1}$ configuration between localized 
$4 f^{5}$ states and itinerant $5 d^{1}$ states $[1]$. The experimentally observed increase at low temperatures is attributed to a contamination with $\mathrm{Sm}^{3+}$ ions or other paramagnetic impurities [1,6]. All these results indicate that the Fermi level $E_{\mathrm{F}}$ is pinned within states in the gap and it is still an open question, whether these in-gap states are extrinsic, coming from imperfections and impurities, or of intrinsic nature.

In the present work we have measured the electrical resistivity and the magnetization of three $\mathrm{SmB}_{6}$ single crystals (one of them is an isotopically enriched ${ }^{154} \mathrm{Sm}^{11} \mathrm{~B}_{6}$ ) with the aim to investigate the properties of the in-gap states which should be responsible for the observed dependences at lower temperatures.

The single crystalline $\mathrm{SmB}_{6}$ samples were prepared by the floating zone melting technique. The resistivity measurements between $80 \mathrm{mK}$ and $3 \mathrm{~K}$ were carried out in a dilution refrigerator, between $1.6 \mathrm{~K}$ and $300 \mathrm{~K}$ in a ${ }^{4} \mathrm{He}$ cryostat. The resistance of the samples located in a dilution refrigerator was measured by an ac resistance bridge, the dc resistance in a ${ }^{4} \mathrm{He}$ cryostat was determined using a $\mathrm{dc}$ nanovoltmeter and a dc current source. The static magnetization between $2 \mathrm{~K}$ and $300 \mathrm{~K}$ was measured in a SQUID magnetometer.

The temperature dependence of the electrical resistivity of all $\mathrm{SmB}_{6}$ samples is shown in Fig. 1. Below $50 \mathrm{~K}$ the resistivity increases nearly exponentially, and between $15 \mathrm{~K}$ and $5 \mathrm{~K}$ it can be approximated by $\rho(T) \propto \exp \left(T_{0} / T\right)$ with $T_{0}^{(1)} \approx 41 \mathrm{~K}$ for sample $1, T_{0}^{(2)} \approx 46 \mathrm{~K}$ for sample 2 and $T_{0}^{(3)} \approx 40 \mathrm{~K}$ for the isotopically enriched sample (denoted as sample 3 ). The main differences between samples can be seen below $3 \mathrm{~K}$. The behavior of $\rho(T)$ of sample 1 between $3 \mathrm{~K}$ and $0.5 \mathrm{~K}$ is strongly influenced by the activated mechanism $\rho(T) \propto \exp \left(T_{\mathrm{g} 1}^{(1)} / T\right)$ with $T_{\mathrm{g} 1}^{(1)} \approx 2.7 \mathrm{~K}$, and the resistivity below $0.2 \mathrm{~K}$ can be described by $\rho(T) \propto \exp \left(T_{\mathrm{g} 2}^{(1)} / T\right)$ with $T_{\mathrm{g} 2}^{(1)} \approx 5.8 \mathrm{mK}$. These results indicate a fine structure in the energy gap which can consist of two narrow bands at $E_{\mathrm{F}}$. In sample 2 the resistivity between $3 \mathrm{~K}$

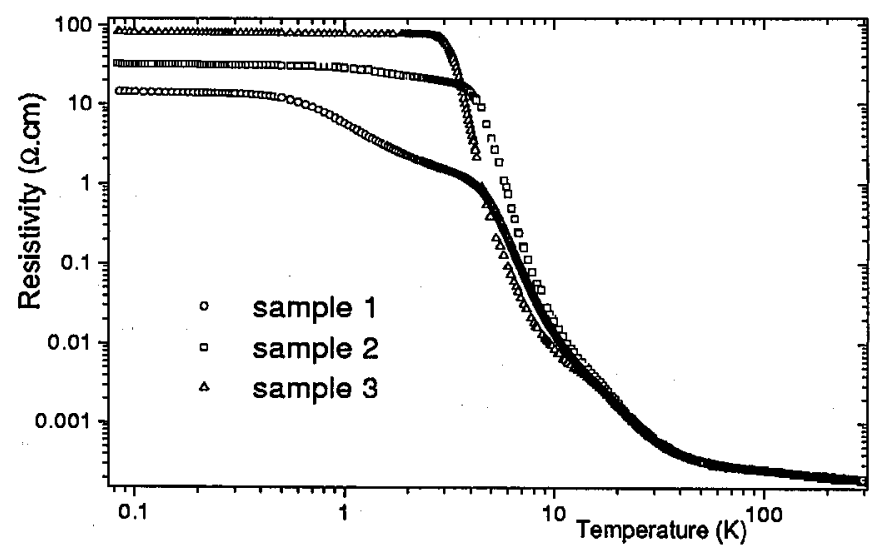

Fig. 1. The temperature dependence of the electrical resistivity of three $\mathrm{SmB}_{6}$ samples. 


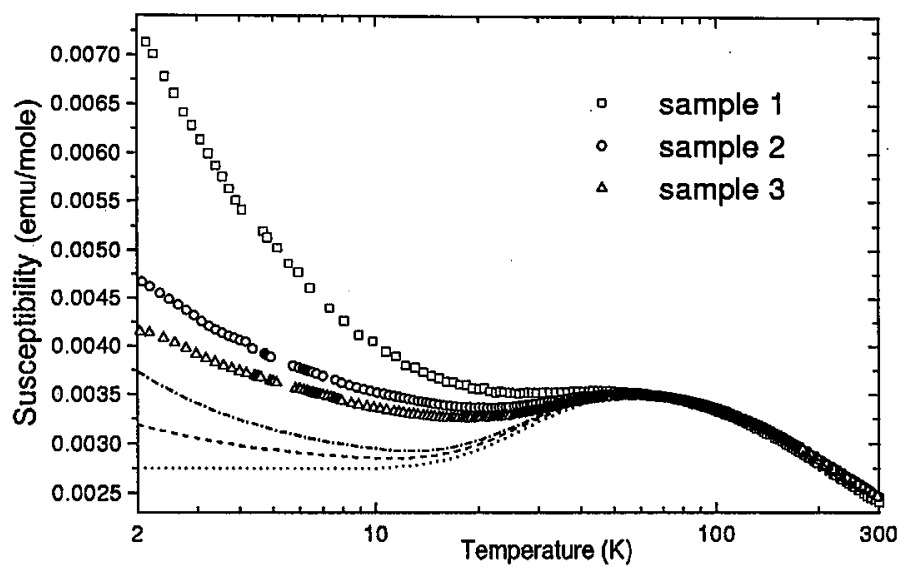

Fig. 2. Magnetic susceptibility of $\mathrm{SmB}_{6}$ samples. Dotted line: van Vleck approximation [1], dashed line: contribution from $1 \%$ of bare $\mathrm{Sm}^{3+}$ ions, dot-and-dash line: contribution from $0.5 \%$ of $4 f^{5} 5 d^{1}$ magnetic in-gap states.

and $0.5 \mathrm{~K}$ fitted by $\rho(T) \propto \exp \left(T_{\mathrm{g}^{1}}^{(2)} / T\right)$ provides $T_{\mathrm{g} 1}^{(2)} \approx 0.8 \mathrm{~K}$, the resistivity at lower temperatures fitted by $\rho(T) \propto \exp \left(T_{\mathrm{g} 2}^{(2)} / T\right)$ provides $T_{\mathrm{g} 2}^{(2)} \approx 3.3 \mathrm{mK}$. For sample 3 no temperature activated term between $3 \mathrm{~K}$ and $0.5 \mathrm{~K}$ was observed. Further, its resistivity below $0.2 \mathrm{~K}$ can be described as $\rho(T) \propto \exp \left(T_{\mathrm{g} 2}^{(3)} / T\right)$ with $T_{\mathrm{g} 2}^{(3)} \approx 3.6 \mathrm{mK}$. Thus all resistivity dependences below $3 \mathrm{~K}$ provide results which are slightly different, however, all samples exhibit activated behavior with an activation energy of a few $\mathrm{mK}$ at lower temperatures. Similar results have been received in Ref. [8], where between $0.9 \mathrm{~K}$ and $40 \mathrm{mK}$ an activation energy of $3 \mathrm{mK}$ was observed. These results indicate that a narrow band separated from the valence or conduction band by an energy gap $\Delta_{2} \propto T_{\mathrm{g} 2} \approx$ a few $\mathrm{mK}$ can exist at $E_{\mathrm{F}}$ in the gap $\Delta_{0} \propto T_{0} \approx 40 \mathrm{~K}$. As the same arrangement is expected in all samples, it can be supposed that these in-gap states originate from the intrinsic electronic structure of $\mathrm{SmB}_{6}$.Impurities and lattice imperfections, mostly present in samples 1 and 2, form other in-gap states which are responsible for the activated term $\Delta_{1} \propto T_{\mathrm{g} 1} \approx 1 \mathrm{~K}$. Impurities and imperfections can also slightly modify the positions of the intrinsic states and lead to a dispersion of $T_{0}$ and $T_{\mathrm{g} 2}$. An in-gap structure of $\mathrm{SmB}_{6}$ containing intrinsic states was recently reported from theoretical studies of the extended Falicov-Kimball model which includes Coulomb repulsion and hybridization between localized and conduction bands [11].

Additional information about the properties of the in-gap states were received from magnetization measurements. The temperature dependence of the static susceptibility in a magnetic field of $50 \mathrm{mT}$ is shown in Fig. 2. At temperatures below $15 \mathrm{~K}$, where the contribution to susceptibility from the ground state of $\mathrm{SmB}_{6}$ should be temperature independent [1], the susceptibility rise can be fitted supposing a presence of paramagnetic impurities in the investigated samples. For sample 1 , the best fit is obtained with about $780 \mathrm{ppm}$ of magnetic impurities with $g J \approx 9-10.5$, where $g$ is the Lande factor and $J$ the total angular momentum. In sample 2 the amount of impurities of similar properties is about $300 \mathrm{ppm}$ and in 
sample 3 the amount is about $180 \mathrm{ppm}$. The impurities can come from lanthanide elements close to $\mathrm{Sm}$ in the periodic table and their different amounts result from the used starting materials and technologies of preparation.

In order to consider the influence of intrinsic in-gap states, contributions of bare $\mathrm{Sm}^{3+}$ ions ( $4 f^{5}$ states) and of magnetic $4 f^{5} 5 d^{1}$ in-gap states between $4 f^{5}$ core states and the $5 d$ electrons were analyzed. If such states are present in the gap, or if they can be produced from the nonmagnetic ground state by low energy thermal excitations (with an activation energy of a few $\mathrm{mK}$ ), then they are expected to generate an additional Curie-like behavior at low temperatures. The contributions coming from the presence of $1 \%$ of bare $\mathrm{Sm}^{3+}$ ions and from the presence of $0.5 \%$ of magnetic $4 f^{5} 5 d^{1}$ configurations are displayed in Fig. 2, and show that the increase in magnetic susceptibility below $15 \mathrm{~K}$ can be explained by intrinsic in-gap states. A Curie-like behavior of magnetic susceptibility at low temperatures was received also from calculations of the magnetic response of the spin-one-half Falicov-Kimball model [12].

In summary, we find that extrinsic (coming from impurities and imperfections) and intrinsic in-gap states seem to be present in the small insulating gap of $\mathrm{SmB}_{6}$ and determine the temperature dependence of electrical resistivity and magnetic susceptibility at low temperatures.

The work was supported by the Slovak scientific grant agency VEGA, under contract 5144-98, and by the Large Scale Facility Program of the EC. Liquid nitrogen needed for experiments was sponsored by VSŽOcel s.r.o., Košice.

\section{References}

[1] P. Wachter, in: Handbook on the Physies and Chemistry of Rare Earths, Eds. K.A. Gschneidner, Jr., L. Eyring, Vol. 19, North-Holland, Amsterdam 1994, p. 177.

[2] G. Aeppli, Z. Fisk, Comments Condens. Matter Phys. 16, 155 (1992).

[3] T. Kasuya, Europhys. Lett. 26, 283 (1994).

[4] B. Gorshunov, N. Sluchanko, A. Volkov, M. Dressel, G. Knebel, A. Loidl, S. Kunii, Phys. Rev. B 59, 1808 (1999).

[5] I. Batko, P. Farkasovsky, K. Flachbart, E.S. Konovalova, Yu.B. Paderno, Solid State Commun. 88, 405 (1993).

[6] J. Roman, V. Pavlik, K. Flachbart, T. Hermannsdurfer, S. Rehmann, E.S. Konovalova, Y.B. Paderno, Physica B 230-232, 715 (1997).

[7] J.W. Allen, B. Batlogg, P. Wachter, Phys. Rev. B 20, 4807 (1979).

[8] A. Lacerda, J.D. Goetee, G.M. Schmiedeshoff, A. Kebede, J.L. Smith, Czech. J. Phys. 46, S4-1991 (1996).

[9] T. Kasuya, K. Takegahara, T. Fujita, T. Tanaka, E. Bannai, J. Phys. (Paris), Collog. 40, C5-308 (1979).

[10] A. Kebede, M.C. Aronson, C.M. Buford, P.C. Canfield, J.H. Cho, B.R. Coles, J.C. Cooley, J.Y. Coulter, Z. Fisk, J. Goetee, W.L. Hults, A. Lacerda, T.D. McLendon, P. Tiwar, J.L. Smith, Physica B 223-224, 256 (1996).

[11] M. Park, J. Hong, J. Korean Phys. Soc. 33, 480 (1998).

[12] J.K. Freericks, V. Zlatic, Phys. Rev. B 58, 322 (1998). 